Article

\title{
Association between Visceral Adipose Tissue and Non-Alcoholic Steatohepatitis Histology in Patients with Known or Suspected Non-Alcoholic Fatty Liver Disease
}

\author{
Ilkay S. Idilman ${ }^{1,2}$, Hsien Min Low ${ }^{1,3}$, Tolga Gidener ${ }^{1}{ }^{\mathbb{C}}$, Kenneth Philbrick ${ }^{1}$, Taofic Mounajjed ${ }^{4}$, Jiahui Li ${ }^{1}$, \\ Alina M. Allen ${ }^{5}$, Meng Yin ${ }^{1}$ and Sudhakar K. Venkatesh ${ }^{1, *(D)}$ \\ 1 Division of Abdominal Imaging, Department of Radiology, Mayo Clinic, Rochester, MN 55905, USA; \\ ipolater@yahoo.com (I.S.I.); hsien_min_low@ttsh.com.sg (H.M.L.); tolgagidener@gmail.com (T.G.); \\ Philbrick.Kenneth@mayo.edu (K.P.); Li.Jiahui@mayo.edu (J.L.); Yin.Meng@mayo.edu (M.Y.) \\ 2 Department of Radiology, School of Medicine, Hacettepe University, Ankara 06100, Turkey \\ 3 Department of Radiology, Tan Tock Seng Hospital, Singapore 308433, Singapore \\ 4 Division of Anatomic Pathology, Mayo Clinic, Rochester, MN 55905, USA; Mounajjed.Taofic@mayo.edu \\ 5 Division of Gastroenterology and Hepatology, Mayo Clinic, Rochester, MN 55905, USA; allen.alina@mayo.edu \\ * Correspondence: venkatesh.sudhakar@mayo.edu
}

Citation: Idilman, I.S.; Low, H.M.; Gidener, T.; Philbrick, K.; Mounajjed, T.; Li, J.; Allen, A.M.; Yin, M.; Venkatesh, S.K. Association between Visceral Adipose Tissue and Non-Alcoholic Steatohepatitis Histology in Patients with Known or Suspected Non-Alcoholic Fatty Liver Disease. J. Clin. Med. 2021, 10, 2565. https://doi.org/10.3390/jcm10122565

Academic Editors: Emmanuel Andrès and Chandana B. Herath

Received: 12 April 2021

Accepted: 4 June 2021

Published: 10 June 2021

Publisher's Note: MDPI stays neutral with regard to jurisdictional claims in published maps and institutional affiliations.

Copyright: (c) 2021 by the authors. Licensee MDPI, Basel, Switzerland. This article is an open access article distributed under the terms and conditions of the Creative Commons Attribution (CC BY) license (https:/ / creativecommons.org/licenses/by/ $4.0 /)$.

\begin{abstract}
Purpose: To determine the association between visceral adipose tissue (VAT) and proton density fat fraction (PDFF) with magnetic resonance imaging (MRI), and hepatic steatosis (HS), non-alcoholic steatohepatitis (NASH) and hepatic fibrosis (HF) in patients with known or suspected non-alcoholic fatty liver disease (NAFLD). (2) Methods: 135 subjects that had a liver biopsy performed within 3 months (bariatric cohort) or 1 month (NAFLD cohort) of an MRI exam formed the study group. VAT volume was quantified at L2-L3 level on opposed-phase images with signal intensity-based painting using a semi-quantitative software. Liver PDFF and pancreas PDFF were calculated on fat fraction maps. Liver volume (Lvol) and spleen volume (Svol) were also calculated using a semi-automated 3D volume tool available on PACS. A histological analysis was performed by an expert hepatopathologist blinded to imaging findings. (3) Results: The mean Lvol, Svol, liver PDFF, pancreas PDFF and VAT of the study population were $2492.2 \mathrm{~mL}, 381.6 \mathrm{~mL}, 13.2 \%, 12.7 \%$ and $120.6 \mathrm{~mL}$, respectively. VAT showed moderate correlation with liver PDFF $(\mathrm{r}=0.41, p<0.001)$ and weak correlation with Lvol $(\mathrm{r}=0.38, p<0.001)$, Svol $(\mathrm{r}=0.20, p=0.025)$ and pancreas PDFF $\left(\mathrm{r}_{\mathrm{S}}=0.29\right.$, $p=0.001)$. VAT, Lvol and liver PDFF were significantly higher in patients with HS ( $p<0.001)$, NASH $(p<0.05)$ and HF $(p<0.05)$. VAT was also significantly higher in the presence of lobular inflammation $(p=0.019)$ and hepatocyte ballooning $(p=0.001)$. The cut-off VAT volumes for predicting HS, NASH and HF were $101.8 \mathrm{~mL}$ (AUC, 0.7), $111.8 \mathrm{~mL}$ (AUC, 0.64) and $111.6 \mathrm{~mL}$ (AUC, 0.66), respectively. (4) Conclusion: The MRI determined VAT can be used for predicting the presence of HS, NASH and HF in patients with known or suspected NAFLD.
\end{abstract}

Keywords: visceral adipose tissue; subcutaneous adipose tissue; non-alcoholic fatty liver disease; non-alcoholic steatohepatitis; hepatic fibrosis

\section{Introduction}

Excessive adipose tissue in the abdomen, particularly in the visceral compartment, is associated with insulin resistance, hyperglycemia, dyslipidemia, systemic hypertension, pro-thrombotic and pro-inflammatory states [1]. Adipose tissue was thought to be a passive reservoir for energy storage; however, now it is regarded as an endocrine organ as it expresses and secretes a variety of bioactive peptides, known as adipokines [2]. Furthermore, visceral adipose tissue (VAT) has a greater influence on hepatic metabolic function in contrast to subcutaneous adipose tissue (SAT) because of its direct access to the liver via the portal system [2]. 
Non-alcoholic fatty liver disease (NAFLD) is the leading cause of chronic liver disease with an estimated global prevalence of $24 \%$, and is increasing worldwide [3,4]. NAFLD comprises of a spectrum ranging from simple steatosis (SS) only without any inflammation or fibrosis, and non-alcoholic steatohepatitis (NASH) which is associated with hepatocyte injury and inflammation, with or without fibrosis [5]. While there is a lower rate of progression to fibrosis in SS, it is estimated that approximately $20 \%$ of patients with NASH will develop cirrhosis in their lifetime [6]. The diagnosis of NASH is based on the histologic examination with liver biopsy which reveals hepatic steatosis (HS), ballooning of hepatocytes and lobular inflammation [5]. However, liver biopsy is invasive and has several limitations such as pain, sampling error and complications including bleeding, infection, bile leak and possible damage to other organs [7]. In view of these limitations, there is a need for a non-invasive method to detect and distinguish the progressive form, $\mathrm{NASH}$, from the relatively benign course of SS.

Although body mass index (BMI) is an independent predictor of NAFLD, body fat composition, particularly visceral adipose tissue (VAT), is known to have association with HS even in non-obese individuals [8]. Magnetic resonance imaging (MRI) derived proton density fat fraction (PDFF) is a non-invasive biomarker that can evaluate hepatic steatosis and correlate with the histologic grade of fatty change [9]. VAT can be easily measured using anatomic images obtained with a computerized tomography (CT) or MRI. Previous studies have shown association between VAT measured with CT and NASH; however, the results were not conclusive [10-12]. The ability to predict NASH from VAT with MRI is not known. An MRI may be suitable for the evaluation of both liver PDFF and VAT at the same time, which is an advantage over CT where only abdominal fat estimation can be reliably determined. In this study, we aimed to determine the association between VAT and NAFLD features in patients with known or suspected NAFLD.

\section{Materials and Methods}

This retrospective study was approved by the institutional review board at Mayo Clinic, Rochester, MN. Two cohorts from a prospective clinical trial (NCT02565446) were included in this study. The first was the bariatric cohort which consisted of 86 obese patients with suspected NAFLD who underwent an MRI within 3 months before the surgery and an intraoperative liver biopsy. The second was the clinical cohort which included 49 patients with NAFLD and at risk of having NASH evaluated by metabolic risk factors such as diabetes mellitus, dyslipidemia, hypertension and obesity. The clinical cohort underwent an MRI within 1 month before the percutaneous liver biopsy.

Patients' age, sex, height, body weight and BMI were obtained from the clinical notes. Obesity was defined as BMI $\geq 30 \mathrm{~kg} / \mathrm{m}^{2}$ based on World Health Organization (WHO) criteria [13]. Insulin resistance was calculated based on fasting plasma glucose and insulin values by using the following homeostasis model assessment (HOMA) and insulin resistance (IR) method calculation: plasma glucose $(\mathrm{mg} / \mathrm{dL}) \times$ insulin $(\mu \mathrm{g} / \mathrm{mL}) / 405$ in 80 patients [14].

All MRI studies were performed on standard 1.5T clinical liver MRI scanners. All the patients underwent a non-contrast enhanced MRI liver protocol. The MRI sequences acquired included: coronal T2-weighted (T2W) and non-contrast enhanced T1-weighted (T1W) sequences, In- and opposed-phase sequences, iterative decomposition of water and fat with echo asymmetry and a least-squares estimation quantification sequence (IDEALIQ, GE Healthcare). The images produced included water only images, fat only images, proton density fat fraction (PDFF) (which produce fat fraction maps from proton density fat fraction (PDFF) and give the option to evaluate the fat fraction of the tissue) and R2* maps. All the MRI sequences included the entire liver.

\subsection{VAT and Subcutaneous Adipose Tissue (SAT) Estimation}

A single reader measured the VAT and SAT in all subjects. As the study population was comprised of obese subjects, L2-L3 level was chosen for VAT quantification. The SAT 
volume was quantified at T12-L1 as the anterior abdominal wall could not be completely included within the field of view at lower levels in many subjects. Both the VAT and SAT were estimated on opposed phase images using a semi-quantitative software Radiology Informatics Laboratory Contour (RIL-Contour) [15] using signal intensity-based painting of the visceral fat by one reader (Figure 1). The opposed-phase image was chosen as the India-ink artifact between the fat and water interface and helped in the demarcation of the extent of the VAT and SAT. Modifications to the automated regions highlighted by the software for finer adjustments were performed by the reader. The VAT and SAT volumes in the single slice were generated by multiplying area with slice thickness. Both the VAT and SAT volumes were expressed in milliliters $(\mathrm{mL})$. SAT quantification was not possible in 49 patients as the abdominal wall touched the magnetic bore altering the anatomy or was not completely covered within the field of view, thereby limiting the segmentation.

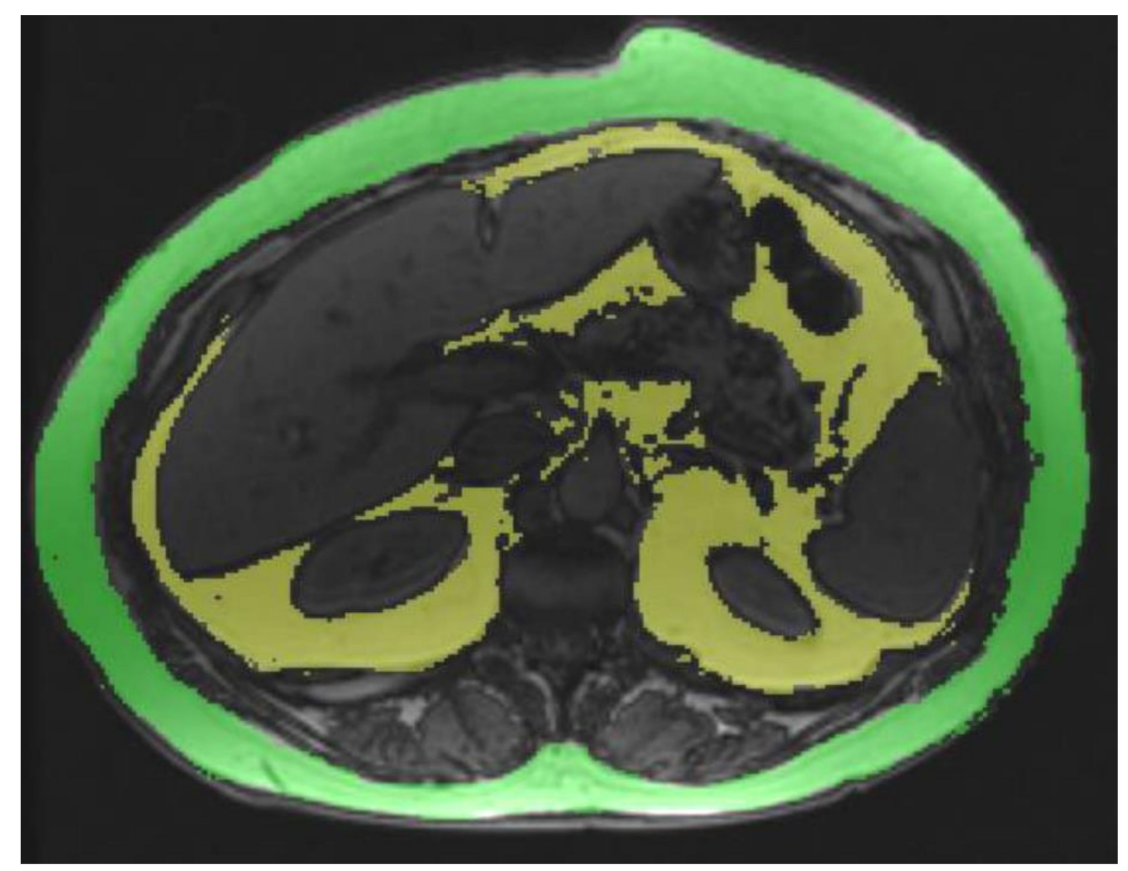

Figure 1. VAT area quantification at L2-L3 level on opposed-phase images using a semi-quantitative software (RIL Contour).

\subsection{Liver and Pancreas PDFF and Liver and Spleen Volumes}

The liver and pancreatic fat fraction was quantified on PDFF images generated from the IDEAL-Q sequence. For each subject, the ROIs were drawn manually in eight anatomic segments by one experienced analyst (J.L) and the mean R2* corrected PDFF was calculated by averaging the values of each ROI and weighted by the area. The pancreas PDFF measurement was also performed by placing the ROIs $\left(>1 \mathrm{~cm}^{2}\right)$ over the head, body and tail of the pancreas and calculating the mean PDFF from the ROIs. All PDFF values were expressed as percentages. One reader (T.G.), blinded to the histological results, independently calculated the liver volume ( $\mathrm{Lvol}$ ) and spleen volume (Svol) on opposed-phase images using a semi-automated 3D volume tool available on PACS (Visage Imaging $\mathrm{GmbH}$, Berlin, Germany).

\subsection{Histological Analysis}

Biopsy specimens were evaluated by an expert hepatopathologist blinded to the clinical and biochemical data (T.M.). Biopsies were scored using the NASH Clinical Research Network (CRN) NAFLD Activity Score (NAS) and fibrosis staging [16]. The pathologist graded hepatic steatosis (grade 0 to 3), lobular inflammation (grade 0 to 3 ) and ballooning [grade 0 to 2] as per NASH CRN guidelines. The NAS score was calculated as previously described [16]. Liver fibrosis staging (stage 0 to 4 ) was also performed. 


\subsection{Statistical Analysis}

Data were summarized as mean $\pm \mathrm{SD}$ or median (minimum maximum) for continuous variables depending on the distributional properties of the data. Normality of the variables was tested by the Kolmogorov-Smirnov test. The Student $t$ test or Mann-Whitney $U$ test was used to assess differences in continuous variables between groups. A one-way ANOVA analysis was performed for comparing VAT in different hepatic steatosis stages. The degree of association between continuous and/or ordinal variables was calculated by using the Pearson correlation coefficient or Spearman's rho. The correlation coefficient $(\rho)$ $>0.7$ was considered strong, 0.4 to 0.7 was considered as moderate and lower than 0.4 was considered weak [17]. Regression analyses were performed to assess the significance of the VAT difference in patients with HS, NASH and HF after correction for age and BMI. A receiver operating curve (ROC) analysis was performed to determine the diagnostic accuracy of VAT and other continuous variables for predicting hepatic steatosis, NASH and fibrosis. Cut-off ranges were calculated using the optimal cut-off to maximize sensitivity and specificity. For all tests, a two-tailed $p$ value of less than 0.05 was considered as statistically significant. All statistical analyses were performed on SPSS (version 22).

\section{Results}

A total of 135 patients (M/F, 94/42) with a mean \pm SD age of $49.2 \pm 11.3$ years were included in this study. The mean \pm SD BMI of the patients was $42.5 \pm 10.1 \mathrm{~kg} / \mathrm{m}^{2}$. The mean \pm SD VAT and SAT were $120.6 \pm 48.6 \mathrm{~mL}$ and $251.6 \pm 73.7 \mathrm{~mL}$, respectively. The mean \pm SD liver PDFF and pancreas PDFF were $13.2 \pm 8.0 \%$ and $12.7 \pm 9.9 \%$, respectively. The mean \pm SD Lvol and Svol were $2492.5 \pm 701.5 \mathrm{~mL}$ and $381.6 \pm 184.4 \mathrm{~mL}$, respectively.

\subsection{Associations among MRI Parameters}

The VAT showed moderate correlation with liver PDFF $(\mathrm{r}=0.41, p<0.001)$ and weak correlation with Lvol $(\mathrm{r}=0.38, p<0.001)$, Svol $(\mathrm{r}=0.20, p=0.025)$ and pancreas PDFF $\left(r_{\mathrm{s}}=0.29, p=0.001\right)$ but no correlation with BMI. The SAT showed strong correlation with BMI $(r=0.78, p<0.001)$, a moderate correlation with Lvol $(\mathrm{r}=0.46, p<0.001)$ and a weak correlation with Svol $(r=0.31, p=0.004)$ but no significant correlation with liver PDFF and pancreas PDFF (Table 1). There was also no correlation between the VAT and SAT $(\mathrm{r}=-0.04, p=0.692)$.

Table 1. Correlation among VAT, SAT, BMI and Lvol, Svol, liver and pancreas PDFF in the study cohort *

\begin{tabular}{cccccc}
\hline & BMI & Lvol & Svol & Liver PDFF & Pancreas PDFF \\
\hline VAT & $\mathrm{r}=0.11(p>0.05)$ & $\mathrm{r}=0.38(p<0.001)$ & $\mathrm{r}=0.19(p=0.025)$ & $\mathrm{r}=0.41(p<0.001)$ & $\mathrm{r}_{\mathrm{S}}=0.29(p=0.001)$ \\
SAT & $\mathrm{r}=0.78(p<0.001)$ & $\mathrm{r}=0.46(p<0.001)$ & $\mathrm{r}=0.31(p=0.004)$ & $\mathrm{r}=0.17(p>0.05)$ & $\mathrm{r}_{\mathrm{S}}=0.18,(p>0.05)$ \\
BMI & & $\mathrm{r}=0.42(p<0.001)$ & $\mathrm{r}=0.27(p=0.002)$ & $\mathrm{r}=0.13(p>0.05)$ & $\mathrm{r}_{\mathrm{S}}=0.24(p=0.005)$ \\
\hline
\end{tabular}

Lvol, liver volume; Svol, spleen volume; VAT, visceral adipose tissue; SAT, subcutaneous adipose tissue. ${ }^{*}=$ The study cohort comprised of 86 bariatric surgery subjects and 49 NAFLD subjects.

There was moderate correlation between liver PDFF and Lvol $(\mathrm{r}=0.61, p<0.001)$ and between liver PDFF and pancreas PDFF $\left(\mathrm{r}_{\mathrm{s}}=0.43, p<0.001\right)$. There was weak but significant correlation between liver PDFF and Svol $(\mathrm{r}=0.21, p=0.05)$. The correlations between pancreas PDFF and BMI $\left(r_{\mathrm{s}}=0.24, p=0.005\right)$, Lvol $\left(\mathrm{r}_{\mathrm{s}}=0.25, p=0.004,\right)$ and HOMA-IR $\left(r_{s}=0.23, p=0.037\right)$ were weak.

\subsection{Histological Analyses}

The histological analysis showed HS in 104 (77\%) patients, NASH in $73(54.1 \%)$ patients and HF in $74(54.8 \%)$ patients. In patients with HS, the ALT $(39.1 \pm 28.1 \mathrm{U} / \mathrm{L}$ vs. $26.9 \pm 18.3 \mathrm{U} / \mathrm{L}, p=0.003)$, triglyceride $(197.1 \pm 95.3 \mathrm{vs} .121 .6 \pm 51.4 \mathrm{mg} / \mathrm{dL}, p<0.001)$, insulin $(44 \pm 52 \mathrm{mcIU} / \mathrm{mL}$ vs. $17.3 \pm 14.2 \mathrm{mcIU} / \mathrm{mL}, p<0.001)$ and HOMA-IR $(13.1 \pm 18.1$ vs. $3.9 \pm 3.5, p<0.001$ ) levels were significantly higher in comparison with patients 
without HS whereas HDL $(39 \pm 8.4 \mathrm{mg} / \mathrm{dL}$ vs. $50.6 \pm 14 \mathrm{mg} / \mathrm{dL}, p=0.001)$ levels were significantly lower.

\subsection{Associations between MRI Parameters and HS, NASH and HF}

The VAT was significantly higher in patients with HS, lobular inflammation, hepatocyte ballooning, NASH and HF (Table 2). Regression analyses demonstrated statistically significant differences in VAT for HS $(p<0.001)$, NASH $(p=0.008)$ and HF $(p<0.001)$ after correction for age and BMI. VAT showed weak correlation with the steatosis grade $(\mathrm{r}=0.31, p<0.001)$, NAS score $(\mathrm{r}=0.28, p=0.001)$ and fibrosis stage $(\mathrm{r}=0.26, p=0.003)$. The differences in VAT according to the fibrosis stage was also significant $(106.6 \pm 46.3 \mathrm{~mL}$, $131.2 \pm 55 \mathrm{~mL}, 142 \pm 48.4 \mathrm{~mL}, 138.9 \pm 45.1$ and $111.2 \pm 23.8 \mathrm{~mL}$ for F0, F1, F2, F3 and F4, respectively, $p=0.012$ ).

With the ROC analysis, the cut-off volumes of VAT were $101.8 \mathrm{~mL}$ (AUC, 0.71 ) for detection of HS, $111.8 \mathrm{~mL}$ (AUC 0.64) for detection of NASH and $111.6 \mathrm{~mL}$ (AUC 0.66) (Table 3) for detection of HF.

Both Lvol and PDFF were significantly higher in patients with HS, NASH and HF (Table 2). Lvol and liver PDFF also showed significant correlation with the steatosis grade $(\mathrm{r}=0.31, p<0.001$ and $\mathrm{r}=0.82, p<0.001)$, NAS score $(\mathrm{r}=0.27, p=0.001$ and $\mathrm{r}=0.71$, $p<0.001)$ and fibrosis stage $(r=0.22, p=0.011$ and $r=0.46, p<0.001$, respectively). Svol was only significantly higher in patients with hepatic fibrosis (Table 2). Svol also had statistically significant correlation with the fibrosis stage $(\mathrm{r}=0.25, p=0.003)$.

There was no significant difference in the SAT in patients with HS, lobular inflammation, hepatocyte ballooning, NASH and HF (Table 2). The SAT did not correlate with the steatosis grade, NAS score or fibrosis stage (Table 4). Interestingly, BMI was lower in patients with HS ( $p=0.04)$ and HF ( $p=0.01)$ with statistical significance (Table 2). There were significant but weak and inverse correlations between the BMI and NAS score $(r=-0.25$, $p=0.003)$ and fibrosis stage $(r=-0.42, p<0.001$, Table 4$)$.

Pancreas PDFF showed a trend to be higher in patients with HS and NASH; however, it was not statistically significant ( 13.3 vs. $10.6 \%$ and $13.9 \%$ vs. $11.4 \%$, respectively). There was a weak but significant correlation between pancreas PDFF and steatosis grade $(r=0.23$, $p=0.007)$. However, there was no correlation between pancreas PDFF and the NAS score and fibrosis stage. There was also no correlation with pancreas PDFF and age $\left(\mathrm{r}_{\mathrm{s}}=0.11, p=0.21\right)$. 


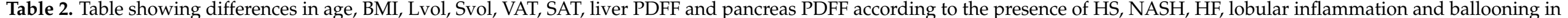
the study cohort ^.

\begin{tabular}{|c|c|c|c|c|c|c|c|c|c|c|c|c|c|c|c|c|}
\hline & \multirow[t]{2}{*}{$\begin{array}{c}\text { Study } \\
\text { Population }\end{array}$} & \multicolumn{2}{|c|}{ Hepatic Steatosis } & \multirow[t]{2}{*}{$p$-Value } & \multicolumn{2}{|c|}{ NASH } & \multirow[t]{2}{*}{$p$-Value } & \multicolumn{2}{|c|}{ Hepatic Fibrosis } & \multirow[t]{2}{*}{$p$-Value } & \multicolumn{2}{|c|}{ Lobular Inflammation } & \multirow[t]{2}{*}{$p$-Value } & \multicolumn{2}{|c|}{ Ballooning } & \multirow[t]{2}{*}{$p$-Value } \\
\hline & & Absent & Present & & Absent & Present & & Absent & Present & & Absent & Present & & Absent & Present & \\
\hline Age (years) & $49.2 \pm 11.2$ & $47.1 \pm 10.5$ & $49.8 \pm 11.4$ & 0.249 * & $47.3 \pm 10.9$ & $50.8 \pm 11.3$ & $0.074^{*}$ & $48.1 \pm 11.5$ & $50.1 \pm 11$ & $0.300^{*}$ & $48.5 \pm 10$ & $49.5 \pm 11.8$ & $0.622^{*}$ & $47.5 \pm 10.5$ & $50.8 \pm 11.7$ & 0.086 * \\
\hline $\operatorname{BMI}\left(\mathrm{kg} / \mathrm{m}^{2}\right)$ & $42.5 \pm 10.1$ & $45.7 \pm 10.5$ & $41.5 \pm 9.8$ & $0.042 *$ & $44.1 \pm 9.5$ & $41.2 \pm 10.5$ & $0.092 *$ & $45.7 \pm 8.9$ & $39.9 \pm 10.3$ & $0.001 *$ & $44.74 \pm 10.17$ & $41.4 \pm 10$ & 0.068 * & $43.9 \pm 10$ & $41.2 \pm 10.1$ & 0.126 * \\
\hline $\begin{array}{l}\text { Lvol (mL) } \\
\text { Svel(mL) }\end{array}$ & $\begin{array}{l}2492.2 \pm 701.5 \\
3816+1844\end{array}$ & $\begin{array}{l}2098.8 \pm 505.9 \\
3298+1596\end{array}$ & $\begin{array}{l}2609.8 \pm 710.7 \\
391 \pm 1892\end{array}$ & $<0.001 *$ & $2312.4 \pm 577.2$ & $2645.4 \pm 762.9$ & $0.005 *$ & $2285.8 \pm 568.3$ & $2662.9 \pm 756.7$ & $0.001 *$ & $2329.25 \pm 590.49$ & $2574.1 \pm 740.4$ & $0.056 *$ & $2304.3 \pm 573.4$ & $2672.4 \pm 766.9$ & $0.002 *$ \\
\hline $\begin{array}{l}\mathrm{VAT}(\mathrm{mL}) \\
\mathrm{SAT}(\mathrm{mL})\end{array}$ & $\begin{array}{l}120.6 \pm \pm 48.6 \\
251.6 \pm 73.7\end{array}$ & $\begin{array}{l}94.3 \pm 40.8 \\
259.1 \pm 70\end{array}$ & $\begin{array}{l}128.5 \pm 48.1 \\
248.5 \pm 75.5\end{array}$ & $\begin{array}{l}<0.000^{*} \\
0.550^{*}\end{array}$ & 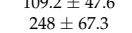 & $\begin{array}{l}130.4 \pm 4.5 \\
2566+82.5\end{array}$ & $\begin{array}{l}0.011)^{*} \\
0.599 *\end{array}$ & $\begin{array}{c}10.66 \text { 4 } 46.3 \\
250 \pm 68\end{array}$ & $\begin{array}{l}132.3 \pm 4.6 \\
254.4+83.5\end{array}$ & $\begin{array}{l}0.0022^{*} \\
0.788^{*}\end{array}$ & $\begin{array}{l}106.8 \pm 49.1 \\
249.9 \pm 70.2\end{array}$ & $\begin{array}{l}127.6 \pm 47 \\
2529+77\end{array}$ & $\begin{array}{l}0.0199^{*} \\
0.849^{*}\end{array}$ & $\begin{array}{l}1482+707.0 \\
2482\end{array}$ & & $0.0608^{*}$ \\
\hline Liver PDFF (\%) & $13.2 \pm 8$ & $5.5 \pm 2$ & $16.3 \pm 7.4$ & $<0.001 *$ & $9.72 \pm 6.5$ & $18 \pm 7.4$ & $<0.001 *$ & $10.25 \pm 6.7$ & $18.11 \pm 7.7$ & $<0.001 *$ & $\begin{array}{r}9.3 \pm 5.6 \\
9.6\end{array}$ & $16.1 \pm 8.4$ & $<0.001$ * & $91 \pm 6.7$ & $17.8 \pm 7.6$ & $<0.001 *$ \\
\hline Pancreas PDFF $(\%)$ & $12.7 \pm 9.9$ & $10.6 \pm 10$ & $13.3 \pm 9.9$ & $0.066 * *$ & $11.4 \pm 8.9$ & $13.9 \pm 10.6$ & $0.195^{* * 4}$ & $14 \pm 11.4$ & $11.6 \pm 8.4$ & 0.322 ** & $11.8 \pm 9.6$ & $13.2 \pm 10.1$ & $0.403^{* *}$ & $12.3 \pm 10.3$ & $13.1 \pm 9.7$ & $0.350 * *$ \\
\hline
\end{tabular}

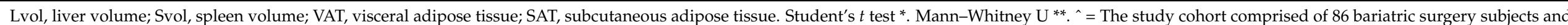
49 NAFLD subjects. 
Table 3. Diagnostic accuracy of VAT for estimation of hepatic steatosis, NASH and hepatic fibrosis in the study cohort *

\begin{tabular}{ccccccc}
\hline & Cut-Off Value & AUC & Sensitivity & Specificity & PPV & NPV \\
\hline Hepatic Steatosis & $101.8 \mathrm{~mL}$ & $0.71(0.60,0.82)$ & $0.70(0.60,0.78)$ & $0.68(0.49,0.83)$ & $0.88(0.78,0.94)$ & $0.40(0.27,0.55)$ \\
NASH & $111.8 \mathrm{~mL}$ & $0.64(0.54,0.73)$ & $0.61(0.49,0.72)$ & $0.65(0.51,0.76)$ & $0.67(0.54,0.78)$ & $0.59(0.46,0.70)$ \\
Hepatic fibrosis & $111.6 \mathrm{~mL}$ & $0.66(0.57,0.76)$ & $0.62(0.50,0.73)$ & $0.64(0.51,0.76)$ & $0.67(0.55,0.78)$ & $0.58(0.46,0.70)$ \\
\hline
\end{tabular}

Numbers in parentheses are $95 \%$ confidence interval. AUC, area under the curve; NPV, negative predictive value; PPV, positive predictive value. ${ }^{*}=$ The study cohort comprised of 86 bariatric surgery subjects and 49 NAFLD subjects.

Table 4. Correlation coefficients among steatosis grade, NAS score, fibrosis stage and BMI, Lvol, Svol, VAT, SAT, liver and pancreas PDFF in the study cohort*.

\begin{tabular}{ccccccc}
\hline Parameter & \multicolumn{2}{c}{ Steatosis Grade } & \multicolumn{2}{c}{ NAS Score } & \multicolumn{2}{c}{ Fibrosis Stage } \\
\hline & $\mathrm{r}_{\mathrm{s}}$ & $p$ & $\mathrm{r}_{\mathrm{s}}$ & $p$ & $\mathrm{r}_{\mathrm{s}}$ & $p$ \\
\hline BMI & -0.17 & 0.053 & -0.25 & 0.003 & -0.42 & $<0.001$ \\
\hline Lvol & 0.31 & $<0.001$ & 0.27 & 0.001 & 0.22 & 0.011 \\
\hline Svol & 0.14 & 0.100 & 0.13 & 0.127 & 0.25 & 0.003 \\
\hline VAT & 0.31 & $<0.001$ & 0.28 & 0.001 & 0.26 & 0.003 \\
\hline SAT & 0.04 & 0.704 & 0.00 & 0.977 & 0.01 & 0.951 \\
\hline Liver PDFF (\%) & 0.82 & $<0.001$ & 0.71 & $<0.001$ & 0.46 & $<0.001$ \\
\hline Pancreas PDFF $(\%)$ & 0.23 & 0.007 & 0.17 & 0.058 & -0.06 & 0.526
\end{tabular}

Lvol, liver volume; Svol, spleen volume; VAT, visceral adipose tissue; SAT, subcutaneous adipose tissue. ${ }^{*}=$ The study cohort comprised of 86 bariatric surgery subjects and 49 NAFLD subjects.

\section{Discussion}

We demonstrated that the VAT has moderate correlation with liver PDFF and weak correlation with Lvol, Svol and pancreas PDFF. In addition, there was weak correlation between VAT and the histological steatosis grade, NAS score and fibrosis stage. The study also showed that VAT has moderate to good discriminative ability for HS, NASH and HF. These findings suggest that VAT has a moderate but definite association with NAFLD changes in the liver. The SAT, however, did not show similar association with NAFLD changes but correlated with BMI and liver volume. Interestingly, BMI was lower in patients with hepatic fibrosis. We also demonstrated significant correlations between liver MRI-PDFF and the hepatic steatosis grade and NAS score.

Choudhary et al. evaluated the VAT and SAT with histologic parameters in a limited number of NAFLD patients $(n=21)$ [10]. They observed that SAT volume correlated significantly with hepatic steatosis; however, none of the adipose tissue volumes had any correlation with other histological variables such as lobular inflammation, ballooning and fibrosis. Yu et al. also demonstrated higher VAT in patients with NAFLD, NASH and significant fibrosis in a total of 324 NAFLD patients and 132 controls, in accordance with our study results [11]. The VAT was, however, derived from single slice computed tomography (CT). Kim et al. demonstrated that a higher VAT area is longitudinally associated with a higher risk of NAFLD, and the baseline SAT area was significantly higher in the subjects who experienced regression of their NAFLD than in the subjects who did not experience regression, regardless of their baseline VAT area [12]. One of the main findings of our study was strong correlations among liver PDFF and hepatic steatosis and the NAS score. In a previous study by Wildman-Tobriner et al. that evaluated 370 patients, strong and moderate correlations were observed between liver PDFF and hepatic steatosis and the NAS score [18].

In the situation of increased insulin resistance, it is hypothesized that pancreatic beta cells produce more insulin to meet the demand which results in beta cell apoptosis 
and consequent increase of adipose tissues [19]. There are many studies evaluating the metabolic effects of pancreatic fat in the literature [20-23]. Patients with higher HOMA-IR had higher pancreatic and liver fat in a study by Patel et al. [20]. Idilman et al. observed higher pancreatic fat in patients with diabetes mellitus in an NAFLD population [21]. Sharma et al. demonstrated higher pancreatic fat fractions in the head and body/tail in type 2 diabetes mellitus patients compared to healthy cohorts [22]. In the present study, we also observed statistically significant but weak correlations between pancreas PDFF and HOMA-IR. However, Kuhn et al. did not observe a significant difference in pancreatic fat in patients with normal glucose tolerance, prediabetes and type 2 diabetes [23]. Patel et al. observed that the presence of hepatic fibrosis has an adverse effect on pancreatic fat and pancreatic fat content was lower in patients with histology-determined liver fibrosis than in those without fibrosis [24]. In our study population, patients with hepatic fibrosis had less pancreas PDFF but results were not statistically significant.

There are conflicting studies in the literature about the association between abdominal fat and hepatic fat content [22,25-27]. Sharma et al. observed statistically significant correlations among liver fat fraction, BMI and SAT [22]. Yu et al. demonstrated statistically significant negative correlations between visceral and subcutaneous fat areas and the three indices of the degree of hepatic fatty infiltration on CT including the attenuation value of liver parenchyma, attenuation difference between liver and spleen and attenuation ratio of liver and spleen with better correlation coefficients in the visceral fat area [26]. Chiyanika et al. evaluated 52 adolescents and observed statistically significant correlations between BMI and SAT and VAT with a better $r$ value for SAT [27]. In their study, they also observed statistically significant correlations between hepatic fat content and SAT and BMI [26]. We also observed a strong correlation between SAT and BMI. However, we did not find a statistically significant correlation between liver PDFF and BMI or SAT. Chiyanika et al. observed a correlation between BMI and pancreatic fat content in accordance with our study. However, Sharma et al. did not demonstrate a correlation between BMI and pancreatic fat content. These differences in study results likely result from the different populations studied.

There are some limitations in the present study. First, we were not able to analyze the SAT in all patients as the abdominal wall could not be completely imaged within the field of view. We also used T12-L1 as the level for determination of SAT as there was anatomic distortion or exclusion of the abdominal wall in the field of view due to patients' obesity. It would have been ideal to measure the VAT and SAT at the same level; however, as our study population was obese it was not possible. However, our findings with SAT are similar to the results reported in the previous literature. Future studies with an MRI on patients without extreme obesity would be useful to see if there are any correlations. We used L2-3 level for the VAT estimation, and this is a standard level for measurement of both VAT and SAT.

Second, there was an interval of up to 3 months between the MRI study and liver biopsy which can affect correlation between MRI findings and histology. The duration may be significant enough for some changes to occur in the pathological features including the degree of hepatic steatosis, degree of inflammation and to a lesser extent stage of fibrosis to occur and potentially affect the correlation analysis. This was unavoidable because scheduling of the MRI and surgeries were dependent on several factors. Third, we did not compare the parameters with a control group of healthy subjects. Our inclusion criteria were to include those with histological results available and it was not feasible to perform invasive liver biopsies without clinical indication. Finally, this is a cross-sectional study with no follow-up data. Future studies are required for assessing the longitudinal changes in the analyzed parameters after the surgery.

In conclusion, VAT volume is associated with NAFLD and may be useful for predicting the presence of HS, NASH and HF in patients with known or suspected NAFLD. 
Author Contributions: Conceptualization, I.S.I. and S.K.V.; methodology, I.S.I., A.M.A., K.P., M.Y., and S.K.V.; software, K.P.; validation, I.S.I., H.M.L., T.G., J.L. and M.Y.; formal analysis, I.S.I., H.M.L., T.G., T.M., J.L. and M.Y.; investigation, I.S.I. and S.K.V.; resources, A.M.A. and S.K.V.; data curation, S.K.V.; writing—original draft preparation, I.S.I. and S.K.V.; writing—review and editing, I.S.I., H.M.L., T.G., K.P., T.M., J.L., A.M.A., M.Y. and S.K.V.; visualization, I.S.I. and S.K.V.; supervision, A.M.A. and S.K.V.; project administration, A.M.A. and S.K.V.; funding acquisition, A.M.A. All authors have read and agreed to the published version of the manuscript (NCT02565446).

Funding: This study was funded by NIH, grant number DK115594.

Institutional Review Board Statement: The study was conducted according to the guidelines of the Declaration of Helsinki, and approved by the Institutional Review Board (IRB code 15-003148) of Mayo Clinic, Rochester, MN.

Informed Consent Statement: Informed consent was obtained from all subjects involved in the study.

Conflicts of Interest: The authors declare no conflict of interest.

\section{References}

1. Grundy, S.M.; Brewer, H.B., Jr.; Cleeman, J.I.; Smith, S.C., Jr.; Lenfant, C. Definition of metabolic syndrome: Report of the National Heart, Lung, and Blood Institute/American Heart Association conference on scientific issues related to definition. Circulation 2004, 109, 433-438. [CrossRef] [PubMed]

2. Kershaw, E.E.; Flier, J.S. Adipose tissue as an endocrine organ. J. Clin. Endocrinol. Metab. 2004, 89, 2548-2556. [CrossRef]

3. Younossi, Z.M.; Koenig, A.B.; Abdelatif, D.; Fazel, Y.; Henry, L.; Wymer, M. Global epidemiology of nonalcoholic fatty liver disease-Meta-analytic assessment of prevalence, incidence, and outcomes. Hepatology 2016, 64, 73-84. [CrossRef]

4. Younossi, Z.; Anstee, Q.M.; Marietti, M.; Hardy, T.; Henry, L.; Eslam, M.; George, J.; Bugianesi, E. Global burden of NAFLD and NASH: Trends, predictions, risk factors and prevention. Nat. Rev. Gastroenterol. Hepatol. 2018, 15, 11-20. [CrossRef] [PubMed]

5. Chalasani, N.; Younossi, Z.; Lavine, J.E.; Charlton, M.; Cusi, K.; Rinella, M.; Harrison, S.A.; Brunt, E.M.; Sanyal, A.J. The diagnosis and management of nonalcoholic fatty liver disease: Practice guidance from the American Association for the Study of Liver Diseases. Hepatology 2018, 67, 328-357. [CrossRef]

6. Matteoni, C.A.; Younossi, Z.M.; Gramlich, T.; Boparai, N.; Liu, Y.C.; McCullough, A.J. Nonalcoholic fatty liver disease: A spectrum of clinical and pathological severity. Gastroenterology 1999, 116, 1413-1419. [CrossRef]

7. Nalbantoglu, I.L.; Brunt, E.M. Role of liver biopsy in nonalcoholic fatty liver disease. World J. Gastroenterol. 2014, 20, 9026-9037. [PubMed]

8. Feng, R.N.; Du, S.S.; Wang, C.; Li, Y.C.; Liu, L.Y.; Guo, F.C.; Sun, C.H. Lean-non-alcoholic fatty liver disease increases risk for metabolic disorders in a normal weight Chinese population. World J. Gastroenterol. 2014, 20, 7932-17940. [CrossRef] [PubMed]

9. Tang, A.; Desai, A.; Hamilton, G.; Wolfson, T.; Gamst, A.; Lam, J.; Clark, L.; Hooker, J.; Chavez, T.; Ang, B.D.; et al. Accuracy of MR imaging-estimated proton density fat fraction for classification of dichotomized histologic steatosis grades in nonalcoholic fatty liver disease. Radiology 2015, 274, 416-425. [CrossRef] [PubMed]

10. Choudhary, N.S.; Duseja, A.; Kalra, N.; Das, A.; Dhiman, R.K.; Chawla, Y.K. Correlation of adipose tissue with liver histology in Asian Indian patients with nonalcoholic fatty liver disease (NAFLD). Ann. Hepatol. 2012, 11, 478-486. [CrossRef] [PubMed]

11. Yu, S.J.; Kim, W.; Kim, D.; Yoon, J.H.; Lee, K.; Kim, J.H.; Cho, E.J.; Lee, J.H.; Kim, H.Y.; Kim, Y.J.; et al. Visceral Obesity Predicts Significant Fibrosis in Patients With Nonalcoholic Fatty Liver Disease. Medicine 2015, 94, e2159. [CrossRef]

12. Kim, D.; Chung, G.E.; Kwak, M.S.; Seo, H.B.; Kang, J.H.; Kim, W.; Kim, Y.J.; Yoon, J.H.; Lee, H.S.; Kim, C.Y. Body Fat Distribution and Risk of Incident and Regressed Nonalcoholic Fatty Liver Disease. Clin. Gastroenterol. Hepatol. 2016, 14, 132-138.e4. [CrossRef] [PubMed]

13. World Health Organization. Report of a WHO Consultation: Definition of Metabolic Syndrome in Definition, Diagnosis and Classification of Diabetes Mellitus; World Health Organization, Department of Noncommunicable Disease Surveillance: Geneva, Switzerland, 1999.

14. Matthews, D.R.; Hosker, J.P.; Rudenski, A.S.; Naylor, B.A.; Treacher, D.F.; Turner, R.C. Homeostasis model assessment: Insulin resistance and beta cell function from fasting plasma glucose and insulin concentrations in man. Diabetologia 1985, 28, 412-419. [CrossRef]

15. Philbrick, K.A.; Weston, A.D.; Akkus, Z.; Kline, T.L.; Korfiatis, P.; Sakinis, T.; Kostandy, P.; Boonrod, A.; Zeinoddini, A.; Takahashi, N.; et al. RIL-Contour: A Medical Imaging Dataset Annotation Tool for and with Deep Learning. J. Digit. Imaging 2019, 32, 571-581. [CrossRef]

16. Kleiner, D.E.; Brunt, E.M.; Van Natta, M.; Behling, C.; Contos, M.J.; Cummings, O.W.; Ferrell, L.D.; Liu, Y.C.; Torbenson, M.S.; Unalp-Arida, A.; et al. Design and validation of a histological scoring system for nonalcoholic fatty liver disease. Hepatology 2005, 41, 1313-1321. [CrossRef] [PubMed]

17. Dancey, C.P.; Reidy, J. Statistics without Maths for Psychology; Pearson Education: Harlow, UK, 2007. 
18. Wildman-Tobriner, B.; Middleton, M.M.; Moylan, C.A.; Rossi, S.; Flores, O.; Chang, Z.A.; Abdelmalek, M.F.; Sirlin, C.B.; Bashir, M.R. Association Between Magnetic Resonance Imaging-Proton Density Fat Fraction and Liver Histology Features in Patients With Nonalcoholic Fatty Liver Disease or Nonalcoholic Steatohepatitis. Gastroenterology 2018, 155, 1428-1435.e2. [CrossRef] [PubMed]

19. Patel, N.S.; Peterson, M.R.; Lin, G.Y.; Feldstein, A.; Schnabl, B.; Bettencourt, R.; Seki, E.; Sirlin, C.B.; Loomba, R. Insulin Resistance Increases MRI-Estimated Pancreatic Fat in Nonalcoholic Fatty Liver Disease and Normal Controls. Gastroenterol. Res. Pract. 2013, 2013, 498296. [CrossRef]

20. Tushuizen, M.E.; Bunck, M.C.; Pouwels, P.J.; Bontemps, S.; Van Waesberghe, J.H.T.; Schindhelm, R.K.; Mari, A.; Heine, R.J.; Diamant, M. Pancreatic fat content and beta-cell function in men with and without type 2 diabetes. Diabetes Care 2007, 30, 2916-2921. [CrossRef] [PubMed]

21. Idilman, I.S.; Tuzun, A.; Savas, B.; Elhan, A.H.; Celik, A.; Idilman, R.; Karcaaltincaba, M. Quantification of liver, pancreas, kidney, and vertebral body MRI-PDFF in non-alcoholic fatty liver disease. Abdom. Imaging 2015, 40, 1512-1519. [CrossRef] [PubMed]

22. Sarma, M.K.; Saucedo, A.; Darwin, C.H.; Felker, E.R.; Umachandran, K.; Kohanghadosh, D.; Xu, E.; Raman, S.; Thomas, M.A. Noninvasive assessment of abdominal adipose tissues and quantification of hepatic and pancreatic fat fractions in type 2 diabetes mellitus. Magn. Reson. Imaging 2020, 72, 95-102. [CrossRef]

23. Kühn, J.P.; Berthold, F.; Mayerle, J.; Völzke, H.; Reeder, S.B.; Rathmann, W.; Lerch, M.M.; Hosten, N.; Hegenscheid, K.; Meffert, P.J. Pancreatic Steatosis Demonstrated at MR Imaging in the General Population: Clinical Relevance. Radiology 2015, 276, 129-136. [CrossRef] [PubMed]

24. Patel, N.S.; Peterson, M.R.; Brenner, D.A.; Heba, E.; Sirlin, C.; Loomba, R. Association between novel MRI-estimated pancreatic fat and liver histology-determined steatosis and fibrosis in non-alcoholic fatty liver disease. Aliment. Pharmacol. Ther. 2013, 37, 630-639. [CrossRef]

25. Jakobsen, M.U.; Berentzen, T.; Sørensen, T.I.; Overvad, K. Abdominal obesity and fatty liver. Epidemiol. Rev. 2007, 29, 77-87. [CrossRef] [PubMed]

26. Yu, A.H.; Duan-Mu, Y.Y.; Zhang, Y.; Wang, L.; Guo, Z.; Yu, Y.Q.; Wang, Y.S.; Cheng, X.G. Correlation between Non-Alcoholic Fatty Liver Disease and Visceral Adipose Tissue in Non-Obese Chinese Adults: A CT Evaluation. Korean J. Radiol. 2018, 19, 923-929. [CrossRef] [PubMed]

27. Chiyanika, C.; Chan, D.F.Y.; Hui, S.C.N.; So, H.K.; Deng, M.; Yeung, D.K.W.; Nelson, E.A.S.; Chu, W.C.W. The relationship between pancreas steatosis and the risk of metabolic syndrome and insulin resistance in Chinese adolescents with concurrent obesity and non-alcoholic fatty liver disease. Pediatr. Obes. 2020, 15, e12653. [CrossRef] [PubMed] 